\title{
A norbornyl route to aminocyclohexitols: syntheses of diverse aminocarbasugars and 'confused' aminocarbasugars
}

\author{
Goverdhan Mehta,* Sripada Lakshminath and Pinaki Talukdar \\ Department of Organic Chemistry, Indian Institute of Science, Bangalore 560 012, India
}

\begin{abstract}
New syntheses of a range of aminocyclitols, of the aminocarbasugar and 'confused' aminocarbasugar type, from simple, readily available norbornyl derived building-blocks is reported. Our synthetic approaches incorporate features that provide for large product diversity in terms of the placement of the amino group on the cyclohexitol matrix.
\end{abstract}

Structural entities having the cyclohexitol (polyhydroxylated cyclohexanoids) or amino cyclohexitol core have aroused widespread synthetic interest because of the diverse biological activity exhibited by them ranging from glycosidase inhibition to mediation of cellular communication. ${ }^{1}$ Carbasugars (also known as pseudosugars) like 1, which has been known since $1966,{ }^{2 a}$ are an important subclass among cyclohexitols which were projected and then proved to be potent glycomimics. While synthetic interest in carbasugars has remained undiminished during the past four decades, ${ }^{1,2}$ a search for their more potent siblings has led to aminocarbasugars from Nature as well as through syntheses. ${ }^{3,4}$ Among the better-known examples of naturally occurring aminocarbasugars are vali- aminocarbasugars have been synthesized in recent years and subjected to biological evaluation and interest in these entities remains unabated. ${ }^{4}$

We have recently delineated ${ }^{5-d}$ general and versatile synthetic approaches based on the bicyclo[2.2.1]heptane (norbornyl) system towards carbasugars 1 and their close siblings, the hitherto unknown 'confused' carbasugars 5 (swapping of the para hydroxymethyl and hydroxy groups of 1) ${ }^{5 \mathrm{~d}}$ This ready access to $\mathbf{1}$ and $\mathbf{5}$ through flexible routes that provide considerable latitude in terms of functional group maneuverability opened opportunity to access a range of new aminocarbasugars and 'confused' aminocarbasugars and these endeavors form the theme of this letter.<smiles>OCC1CC(O)C(O)C(O)C1O</smiles>

1<smiles>N[C@H]1C[C@H](CO)[C@@H](O)[C@H](O)[C@H]1O</smiles>

2<smiles>N[C@H]1C=C(CO)[C@@H](O)[C@H](O)[C@H]1O</smiles>

3<smiles>N[C@H]1C[C@](O)(CO)[C@@H](O)[C@H](O)[C@H]1O</smiles>

4<smiles>OCC1C(O)CC(O)C(O)C1O</smiles>

5 damine $2{ }^{3 a}$ valienamine $\mathbf{3}$ and valiolamine $\mathbf{4}$ and some of these and their derivatives have found commercial use. In aminocarbasugars 2-4, the amino group is located on C-1. However, replacement of any of the other hydroxy groups by an amino group on the carbasugar platform 1 offers vast opportunities for structural and stereochemical variation, a point not missed by the enterprising synthetic community. As a result, many new types of

*Corresponding author. Fax: +91-80-3600936; e-mail: gm@ orgchem.iisc.ernet.in
Our first foray towards aminocarbasugars and 'confused' aminocarbasugars emanated from the readily available 7-norbornanone derivative $\mathbf{6}$, which undergoes smooth fragmentation to functionalized cyclohexanoid $\mathbf{7}$, as recently reported by us. ${ }^{5 b}$ Elaboration of $\mathbf{7}$ to $\mathbf{8}$ was straightforward and further catalytic aminohydroxylation $^{6}$ proceeded stereoselectively and with good regioselectivity to furnish a mixture (4:1) of $\mathbf{9}$ and $\mathbf{1 0}$ (Scheme 1). ${ }^{7}$ Reductive $N$-detosylation and removal of the protective groups in $\mathbf{9}$ and $\mathbf{1 0}$ led to $\mathbf{1 1}$ and 12, respectively (Scheme 1). ${ }^{7}$ While 11 turned out to be carbagalactovalidamine, whose synthesis has been described by two 

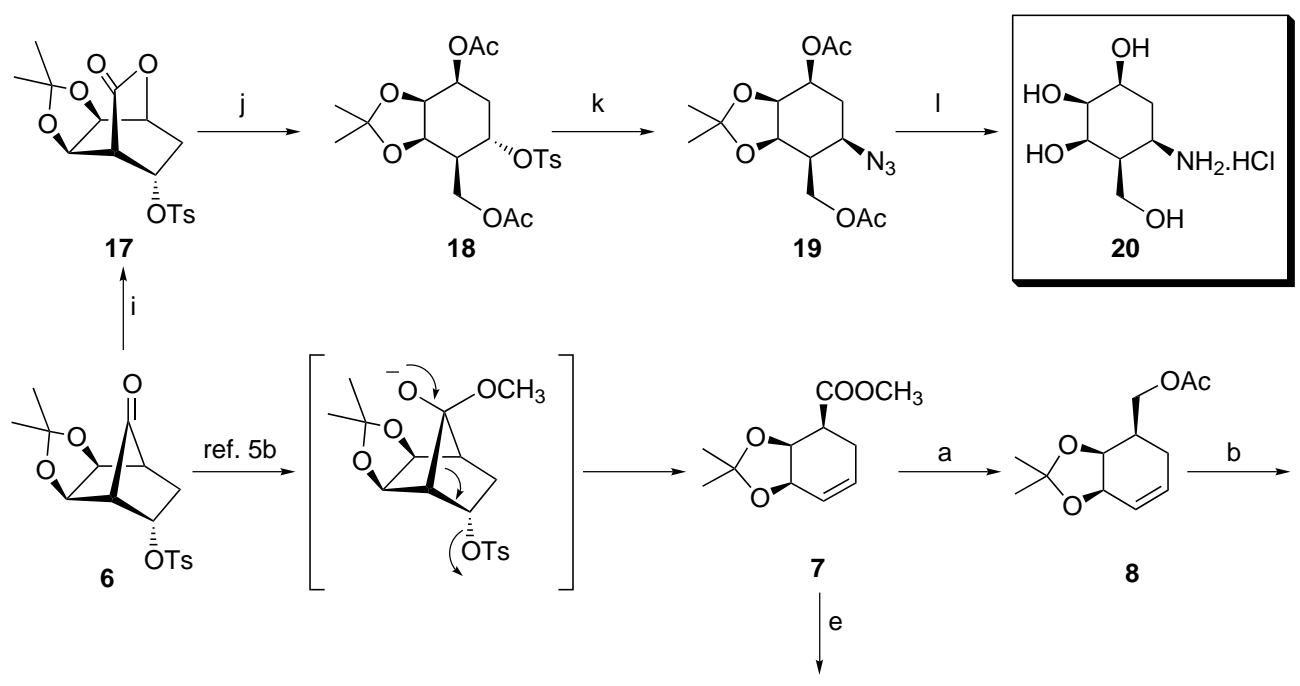

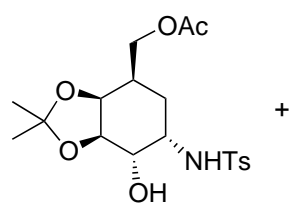

9
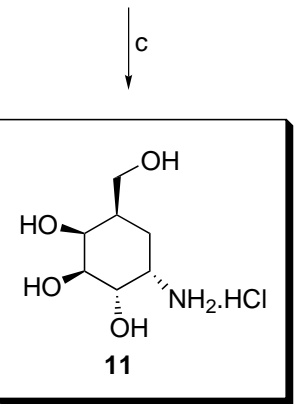

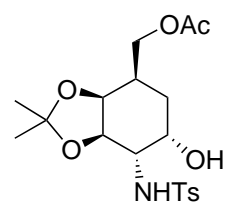

10
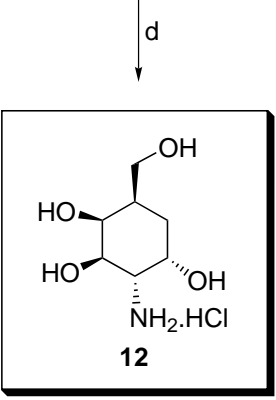

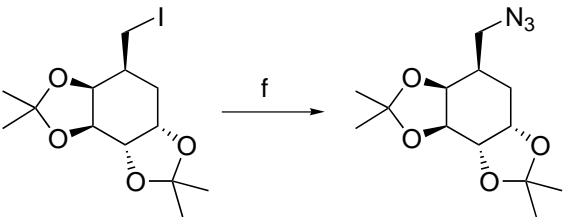

13

14

Scheme 1. Reagents and conditions: (a) Ref. 5b; (b) Chloramine-T, $\mathrm{OsO}_{4},{ }^{t} \mathrm{BuOH}_{-} \mathrm{H}_{2} \mathrm{O}$ (4:1), rt, 12 h, 70\% (9:10; 4:1); (c) i. Ac ${ }_{2} \mathrm{O}$, DMAP, DCM, rt, 2 h, 95\%, ii. Na-Naphthalenide, DME, $-60^{\circ} \mathrm{C}, 4$ h, 65\%, iii. $1 \mathrm{~N} \mathrm{HCl}, \Delta, 20$ h, $90 \%$; (d) i. Ac 2 , DMAP, DCM, rt, 2 h, 93\%, ii. Na-Naphthalenide, DME, $-60^{\circ} \mathrm{C}, 4$ h, 45\%, iii. $1 \mathrm{~N} \mathrm{HCl}, \Delta, 20$ h, quant.; (e) Ref. $5 \mathrm{c}$; (f) $\mathrm{NaN}, \mathrm{DMF}_{3}, 100^{\circ} \mathrm{C}, 5$ h, 92\%; (g) i. $\mathrm{H}_{2}, \mathrm{Pd} / \mathrm{CaCO}_{3}, \mathrm{EtOH}, \mathrm{rt}, 3$ h, ii. Ac $2 \mathrm{O}$, DMAP, DCM, rt, 60\% (two steps); (h) $1 \mathrm{~N} \mathrm{HCl,} \Delta, 20$ h, $92 \%$; (i) $\mathrm{MCPBA}$, $\mathrm{Na}_{2} \mathrm{CO}_{3}$, DCM, rt, 4 h, $85 \%$ (87:13 regioisomeric mixture of lactones); (j) i. LAH, THF, $0{ }^{\circ} \mathrm{C}-\mathrm{rt}, 2 \mathrm{~h}, 85 \%$, ii. Ac $2 \mathrm{O}, \mathrm{DMAP}$, DCM, rt, 92\%; (k) $\mathrm{NaN}_{3}, \mathrm{DMF}, 90^{\circ} \mathrm{C}, 5 \mathrm{~h}, 82 \%$; (1) i. $\mathrm{H}_{2}, \mathrm{Pd} / \mathrm{CaCO}_{3}, \mathrm{EtOH}, \mathrm{rt}, 3 \mathrm{~h}, 80 \%$, ii. $1 \mathrm{~N} \mathrm{HCl}, \Delta, 25 \mathrm{~h}, 90 \%$.

groups recently, ${ }^{4 \mathrm{~h}, \mathrm{k}}$ the minor aminocarbasugar $\mathbf{1 2}$ is a new entity. A new carbasugar with an amino group in the side arm could also be accessed from the versatile precursor 7 through conversion to the iodomethyl derivative $\mathbf{1 3}$ and azide ion-mediated displacement to 14. Catalytic hydrogenation to the amine functionality, characterized as amide $\mathbf{1 5}$ and deprotection furnished the new carbagalactosamine 16 (Scheme 1). ${ }^{7}$ endoKeto-tosylate $\mathbf{6}$ also proved to be an effective precursor for 'confused' aminocarbasugars. Baeyer-Villiger oxidation of 6 was regioselective $(87: 13)$ and lactone 17 was the dominant product (Scheme 1). $\mathrm{LiAlH}_{4}$ reduction and acetylation gave protected cyclohexitol 18. Displacement of the tosylate with azide ion in $\mathbf{1 8}$ was smooth and delivered the azido compound 19 (Scheme 1). Finally, the reduction of the azide functionality in 19 and a deprotection sequence delivered the first 'confused' aminocarbasugar 20 (Scheme 1). ${ }^{7}$
The successful acquisition of $\mathbf{2 0}$ and the inherent flexibility of our simple access to the 'confused' carbasugars presented an opportunity to generate diversity through placement of the amino group at various positions on the cyclohexitol framework. For this purpose, endoketo-acetate 22, obtained ${ }^{5 \mathrm{~d}}$ from abundantly available 21, was found to be most serviceable. Baeyer-Villiger oxidation of $\mathbf{2 2}$ led to the regioisomeric lactones 23 and 24 in which the later predominated (13:87) (Scheme 2). The 'confused' carbasugar monoacetonide derivative 25 obtained from $\mathbf{2 4}$ was subjected to bis-acetonide formation and led to a mixture (43:47:10) of bis-acetonides 26-28, ${ }^{7}$ leaving one hydroxyl group free in each one of them for further manipulation (Scheme 2). The hydroxy group in the two major bis-acetonides 26 and 27 was transformed to the mesylate leaving group and then displaced with azide ion to furnish azido compounds 29 and 30, respectively (Scheme 2). Reduction of the azido 

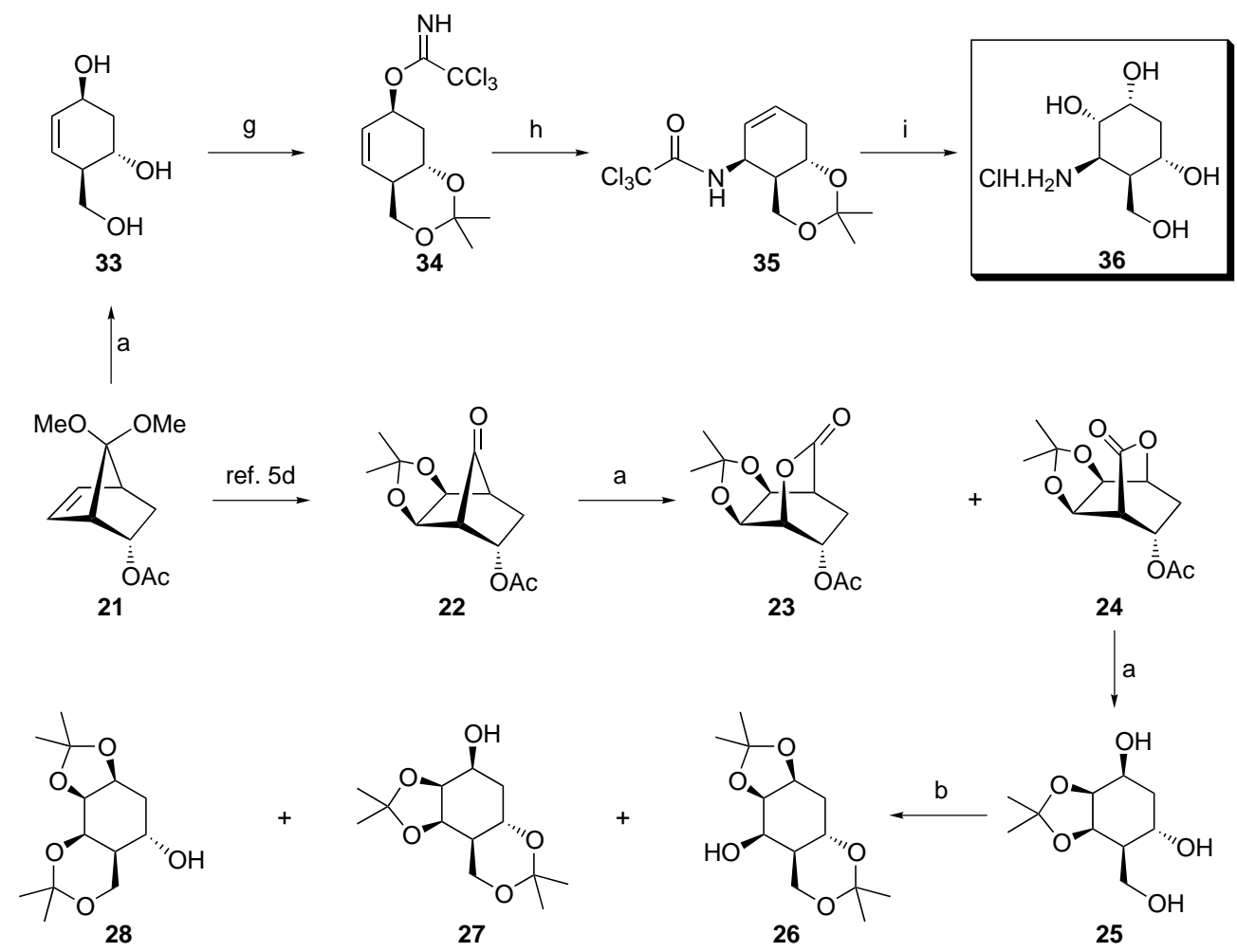<smiles>[Te][Te]</smiles>
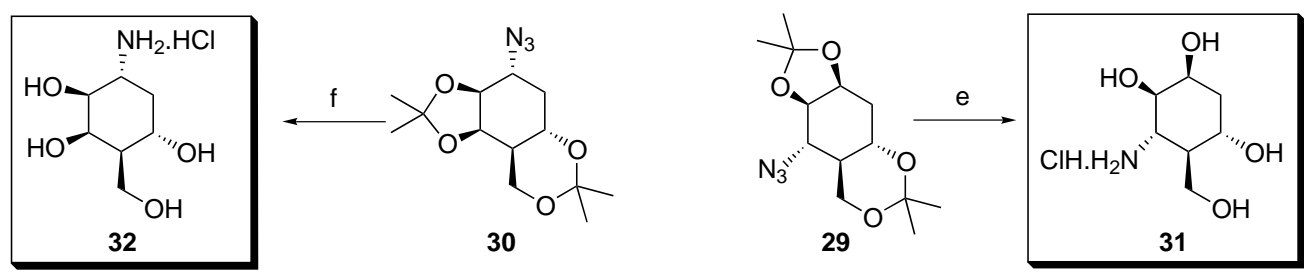

Scheme 2. Reagents and conditions: (a) Ref. 5d; (b) $\mathrm{Me}_{2} \mathrm{CO}$, Amberlyst-15, rt, 2 h, 78\% (26:27:28; 43:47:10); (c) i. MsCl, Py, rt, 10 h, ii. $\mathrm{NaN}_{3}, \mathrm{DMF}, 100^{\circ} \mathrm{C}, 5 \mathrm{~h}, 65 \%$ (two steps); (d) i. $\mathrm{MsCl}_{2} \mathrm{Et}_{3} \mathrm{~N}, \mathrm{DMAP}, \mathrm{DCM}, \mathrm{rt}, 1 \mathrm{~h}$, ii. $\mathrm{NaN}_{3}, \mathrm{DMF}, 100^{\circ} \mathrm{C}, 4 \mathrm{~h}, 70 \%$ (two steps); (e) i. $\mathrm{H}_{2}, \mathrm{Pd} / \mathrm{CaCO}_{3}, \mathrm{EtOH}, \mathrm{rt}, 3 \mathrm{~h}$, ii. $1 \mathrm{~N} \mathrm{HCl}, \Delta, 20 \mathrm{~h}, 80 \%$ (two steps); (f) same as (e), $85 \%$ (two steps); (g) i. $\mathrm{Me}_{2} \mathrm{CO}$, Amberlyst-15, rt, 2 h, $81.5 \%$, ii. $\mathrm{CCl}_{3} \mathrm{CN}, \mathrm{DBU}, 0^{\circ} \mathrm{C}, 1 \mathrm{~h}, 93 \%$; (h) i. $\mathrm{K}_{2} \mathrm{CO}_{3}, p$-xylene, reflux, 12 h, $70 \%$; (i) i. OsO $\mathrm{NMMO}, \mathrm{Me}_{2} \mathrm{CO}-\mathrm{H}_{2} \mathrm{O}-t \mathrm{BuOH}$ (5:5:2), rt, 2 days, 92\%, ii. $1 \mathrm{~N} \mathrm{HCl}, \Delta, 36 \mathrm{~h}$, quant.

group in $\mathbf{2 9}$ and $\mathbf{3 0}$ through catalytic hydrogenation and deprotection delivered the 'confused' aminocarbasugars $31^{7}$ and $32,{ }^{7}$ respectively (Scheme 2 ).

An interesting stereochemical variant could also be added to the design of aminocyclitols from $\mathbf{2 1}$. We have recently shown that $\mathbf{2 1}$ could be readily transformed to the trihydroxy-cyclohexene 33 via regioselective BaeyerVilliger oxidation and hydride reduction. ${ }^{5 \mathrm{~d}}$ Acetonide protection in $\mathbf{3 3}$ and reaction with trichloroacetonitrile led to the allyl trichloroacetimidate $\mathbf{3 4}$ and set the stage for an Overman rearrangement ${ }^{8}$ to allyl trichloroacetamide 35 under thermal activation in the presence of a base (Scheme 2). Catalytic $\mathrm{OsO}_{4}$-mediated dihydroxylation on $\mathbf{3 5}$ was stereoselective and further deprotection led to a stereochemically diverse 'confused' aminocarbasugar 36 . $^{7}$

The availability of so many new and diverse range of aminocyclitols 16, 20, 31, 32 and 36 led us to explore in a preliminary way their glycosidase inhibitory activity against a set of six commonly used enzymes $(\alpha-$ and $\beta$-glucosidase, $\alpha$ - and $\beta$-galactosidase, and $\alpha$ - and $\beta$-mannosidase) following the standard protocol followed by us. ${ }^{9}$ However, we have not observed any notable inhibition to report at this time and clearly more screening is warranted.

In short, we have amplified the utility of the norbornyl building blocks 6 and 22 by obtaining a range of aminocarbasugars and 'confused' aminocarbasugars, the latter as new entities for the first time, through simple, regio- and stereocontrolled operations.

\section{Acknowledgements}

S.L. thanks CSIR for the award of a Research Fellowship. We appreciate the support of this research by the Chemical Biology Unit of the Jawaharlal Nehru Centre 
for Advanced Scientific Research, Bangalore. We thank S.I.F. at I.I.Sc., Bangalore for 2D NMR data.

\section{References}

1. Recent reviews: (a) Suami, T. Top. Curr. Chem. 1990, 154, 257; (b) Ferrier, R. J.; Middleton, S. Chem. Rev. 1993, 93, 2779; (c) Martinez-Grau, A.; Marco-Contelles, J. Chem. Soc. Rev. 1998, 27, 155; (d) Berecibar, A.; Grandjean, C.; Siriwardena, A. Chem. Rev. 1999, 99, 779.

2. Some lead references: (a) McCasland, G. E.; Furuta, S.; Durham, L. J. J. Org. Chem. 1966, 31, 1516; (b) Pingli, L.; Vandewalle, M. Synlett 1994, 228; (c) Hudlicky, T.; Entwistle, D. A.; Pitzer, K. K.; Thorpe, A. J. Chem. Rev. 1996, 96, 1195; (d) Dalko, P. I.; Sinay, P. Angew. Chem., Int. Ed. Engl. 1999, 38, 773.

3. (a) Horii, S.; Iwasa, T.; Mizuta, E.; Kameda, Y. $J$. Antibiot. 1971, 24, 59; (b) Kameda, Y.; Horii, A. Chem. Commun. 1972, 746; (c) Kameda, Y.; Asano, N.; Yoshikawa, M.; Takeuchi, M.; Yamaguchi, T.; Matsui, K.; Horii, S.; Fukase, H. J. Antibiot. 1984, 37, 1701.

4. Selected references directed towards the syntheses of aminocarbasugars: (a) Ogawa, S.; Ito, H.; Ogawa, T.; Iwasaki, S.; Suami, T. Bull. Chem. Soc. Jpn. 1983, 56, 2319; (b) Ogawa, S.; Suzuki, M.; Tonegawa, T. Bull. Chem. Soc. Jpn. 1988, 61, 1824; (c) Ogawa, S.; Taki, T.; Isaka, A. Carbohydr. Res. 1989, 191, 154; (d) Ogawa, S.; Orihara, M. Carbohydr. Res. 1989, 189, 323; (e) Ogawa, S.; Tonegawa, T. Carbohydr. Res. 1990, 204, 51; (f) Acena, J. L.; Arjona, O.; Fernandez de la Pradilla, R.; Plumet, J.; Viso, A. J. Org. Chem. 1994, 59, 6419; (g) Shing, T. K. M.; Tai, V. W.-F. J. Org. Chem. 1995, 60, 5332; (h) Kameda, Y.; Kawashima, K.; Takeuchi, M.; Ikeda, K.; Asano, N.; Matsui, K. Carbohydr. Res. 1997, 300, 259; (i) Afarinkia, K.; Mahmood, F. Tetrahedron 1999, 55, 3129; (j) Rassu, G.; Auzzas, L.; Pinna, L.; Battistini, L.; Zanardi, F.; Marzocchi, L.; Acquotti, D.; Casiraghi, G. J. Org. Chem. 2000, 65, 6307; (k) Ogawa, S.; Sekura, R.; Maruyama, A.; Yuasa, H.; Hashimoto, H. Eur. J. Org. Chem. 2000, 2089.

5. (a) Mehta, G.; Mohal, N. Tetrahedron Lett. 1998, 39, 3285; (b) Mehta, G.; Mohal, N.; Lakshminath, S. Tetrahedron Lett. 2000, 41, 3505; (c) Mehta, G.; Lakshminath, S. Tetrahedron Lett. 2000, 41, 3509; (d) Mehta, G.; Talukdar, P.; Mohal, N. Tetrahedron Lett. 2001, 42, 7663.

6. (a) Li, G.; Sharpless, K. B. Angew. Chem., Int. Ed. Engl.
1996, 35, 451; (b) O'Brien, P. Angew. Chem., Int. Ed. Engl. 1999, 38, 326.

7. All new compounds reported here were racemic and characterized on the basis of spectroscopic data (IR, ${ }^{1} \mathrm{H},{ }^{13} \mathrm{C}$ NMR and mass). Selected spectroscopic data ( $J$ in $\mathrm{Hz}) . \mathbf{1 1}$ : $\delta_{\mathrm{H}}\left(300 \mathrm{MHz}, \mathrm{D}_{2} \mathrm{O}\right): 3.97(1 \mathrm{H}$, br d, $J 2.1), 3.87(1 \mathrm{H}, \mathrm{ddd}$, $J 1.8,4.8,10), 3.61(1 \mathrm{H}, \mathrm{br} \mathrm{s}), 3.51-3.48(2 \mathrm{H}, \mathrm{m}), 3.39(1 \mathrm{H}$, d $1 / 2 \mathrm{ABq}, J 6.3,11), 1.78(1 \mathrm{H}, \mathrm{m}, \mathrm{q}$ like), $1.68-1.65(2 \mathrm{H}$, $\mathrm{m}) ; \delta_{\mathrm{C}}\left(75 \mathrm{MHz}, \mathrm{D}_{2} \mathrm{O}\right): 71.6(\mathrm{CH}), 69.8(\mathrm{CH}), 68.1(\mathrm{CH})$, $62.6\left(\mathrm{CH}_{2}\right), 52.0(\mathrm{CH}), 37.2(\mathrm{CH}), 24.1\left(\mathrm{CH}_{2}\right) .12: \delta_{\mathrm{H}}(300$ $\left.\mathrm{MHz}, \mathrm{D}_{2} \mathrm{O}\right): 4.12(1 \mathrm{H}$, br d, $J$ 3.3), $3.96(1 \mathrm{H}$, br s $), 3.72$ $(1 \mathrm{H}, \mathrm{dd}, J 2.4,10.5), 3.5(1 \mathrm{H}, \mathrm{d} 1 / 2 \mathrm{ABq}, J 8,11), 3.37$ $(1 \mathrm{H}, \mathrm{d} 1 / 2 \mathrm{ABq}, J 6.5,11), 3.26-3.22(1 \mathrm{H}, \mathrm{m}), 1.99-1.87$ $(1 \mathrm{H}, \mathrm{m}), 1.57(1 \mathrm{H}, \mathrm{br}$ td, $J 3.6,14.4), 1.47-1.37(1 \mathrm{H}, \mathrm{m})$; $\delta_{\mathrm{C}}\left(75 \mathrm{MHz}, \mathrm{D}_{2} \mathrm{O}\right): 69.9(\mathrm{CH}), 69.1(\mathrm{CH}), 66.2(\mathrm{CH}), 63.1$ $\left(\mathrm{CH}_{2}\right), 54.4(\mathrm{CH}), 36.8(\mathrm{CH}), 28.7\left(\mathrm{CH}_{2}\right) .16: \delta_{\mathrm{H}}(300$ $\left.\mathrm{MHz}, \mathrm{D}_{2} \mathrm{O}\right): 4.05(1 \mathrm{H}, \mathrm{m}), 3.98(1 \mathrm{H}, \mathrm{br} \mathrm{s}), 3.65(2 \mathrm{H}, \mathrm{m})$, $3.07(1 \mathrm{H}, \mathrm{d} 1 / 2 \mathrm{ABq}, J 7,13), 2.94(1 \mathrm{H}, \mathrm{d} 1 / 2 \mathrm{ABq}, J 7$, 13), $2.21-2.10(1 \mathrm{H}, \mathrm{m}), 1.65-1.61(2 \mathrm{H}, \mathrm{m}) ; \delta_{\mathrm{C}}(75 \mathrm{MHz}$, $\left.\mathrm{D}_{2} \mathrm{O}\right)$ : $71.5(\mathrm{CH}), 71.3(\mathrm{CH}), 71.2(\mathrm{CH}), 69.2(\mathrm{CH}), 42.0$ $\left(\mathrm{CH}_{2}\right), 32.9(\mathrm{CH}), 29.0\left(\mathrm{CH}_{2}\right) .20: \delta_{\mathrm{H}}\left(300 \mathrm{MHz}, \mathrm{D}_{2} \mathrm{O}\right)$ : 3.97-3.91 (3H, m), 3.79-3.69 (2H, m, series of d), 3.39$3.33(1 \mathrm{H}, \mathrm{m}, \mathrm{dd}$ like), $2.11(1 \mathrm{H}, \mathrm{td}, J 6,12), 1.33(1 \mathrm{H}, \mathrm{m}$, q like), $1.45(1 \mathrm{H}, \mathrm{dt}, J 3.3,7.5) ; \delta_{\mathrm{C}}\left(75 \mathrm{MHz}, \mathrm{D}_{2} \mathrm{O}\right): 84.6$ $(\mathrm{CH}), 73.6(\mathrm{CH}), 68.7(\mathrm{CH}), 66.7\left(\mathrm{CH}_{2}\right), 47.9(\mathrm{CH}), 45.1$ $(\mathrm{CH}), 30.9\left(\mathrm{CH}_{2}\right) .31: \delta_{\mathrm{H}}\left(300 \mathrm{MHz}, \mathrm{D}_{2} \mathrm{O}\right): 4.04(1 \mathrm{H}, \mathrm{br} \mathrm{s})$, $3.89(1 \mathrm{H}, \mathrm{d} 1 / 2 \mathrm{ABq}, J 3.3,11.7), 3.78-3.63(3 \mathrm{H}, \mathrm{m}), 3.32$ $(1 \mathrm{H}, \mathrm{t}, J 11), 2.11(1 \mathrm{H}, \mathrm{td}, J 4,13.8), 1.65(1 \mathrm{H}, \mathrm{ddt}, J 3.3$, $7.5,10.8), 1.56(1 \mathrm{H}, \mathrm{dt}, J 2.1,13.8) ; \delta_{\mathrm{C}}\left(75 \mathrm{MHz}, \mathrm{D}_{2} \mathrm{O}\right)$ : $72.2(\mathrm{CH}), 68.9(\mathrm{CH}), 64.5(\mathrm{CH}), 60.7\left(\mathrm{CH}_{2}\right), 53.1(\mathrm{CH})$, $47.4(\mathrm{CH}), 38.5\left(\mathrm{CH}_{2}\right) \cdot 32: \delta_{\mathrm{H}}\left(300 \mathrm{MHz}, \mathrm{D}_{2} \mathrm{O}\right): 4.04(1 \mathrm{H}$, t, $J 2.7), 3.76(1 \mathrm{H}, \mathrm{d} 1 / 2 \mathrm{ABq}, J 4.5,11), 3.66(1 \mathrm{H}, \mathrm{dt}, J$ $4.5,10.8), 3.59(1 \mathrm{H}, \mathrm{d} 1 / 2 \mathrm{ABq}, J 8.4,11.1), 3.53(1 \mathrm{H}, \mathrm{dd}$, $J$ 2.7, 10.8), $3.28(1 \mathrm{H}, \mathrm{m}, \mathrm{dt}$ like), $2.20(1 \mathrm{H}, \mathrm{td}, J 4.5,12)$, $1.59(1 \mathrm{H}$, dddd, $J 2.7,4.5,7,8.4), 1.43(1 \mathrm{H}, \mathrm{q}, J 12) ; \delta_{\mathrm{C}}$ (75 MHz, $\left.\mathrm{D}_{2} \mathrm{O}\right): 72.9,69.8,65.6,60.4,49.8,48.9,36.8 .36$ : $\delta_{\mathrm{H}}\left(300 \mathrm{MHz}, \mathrm{D}_{2} \mathrm{O}\right): 3.9-3.86(2 \mathrm{H}, \mathrm{m}), 3.82(1 \mathrm{H}, \mathrm{dd}, J 3.3$, 8.4), 3.69-3.64 (2H, m), $3.57(1 \mathrm{H}, \mathrm{d} 1 / 2 \mathrm{ABq}, J 5.7,11)$, $2.22-2.19(1 \mathrm{H}, \mathrm{m}), 1.80-1.75(2 \mathrm{H}, \mathrm{m}) ; \delta_{\mathrm{C}}\left(75 \mathrm{MHz}, \mathrm{D}_{2} \mathrm{O}\right)$ : $68.9(\mathrm{CH}), 68.7(\mathrm{CH}), 66.8(\mathrm{CH}), 59.7\left(\mathrm{CH}_{2}\right), 51.6(\mathrm{CH})$, $44.3(\mathrm{CH}), 33.9\left(\mathrm{CH}_{2}\right)$.

8. (a) Overman, L. E. Acc. Chem. Res. 1980, 13, 218; (b) Nishikawa, T.; Asai, M.; Ohyabu, N.; Isobe, M. J. Org. Chem. 1998, 63, 188.

9. (a) Mehta, G.; Ramesh, S. S. Chem. Commun. 2000, 2429; (b) Mehta, G.; Ramesh, S. S. Tetrahedron Lett. 2001, 42, 1987. 